lar transform, rather than some other, is the object of such intensive study. The fact that there exists a general theory of transforms on locally compact abelian groups, which establishes such basic results as the uniqueness theorem for $L_{1}$ transforms, the closure theorems for translations of functions in $L_{1}$ and $L_{2}$, and the generalized Plancherel theorem, in forms which apply to Fourier integrals and series on $n$ dimensional euclidean space, as well as to almost periodic functions, expansions into Walsh-Rademacher functions, functions on finite groups, and so forth, is devoid of any significant recognition.

Thus the book is intensive rather than extensive in scope, and strength of logical organization has been somewhat sacrificed for elegance of technique and mathematical style. It is a really valuable addition to the literature, and substantial numbers of students should find it both pleasant and instructive.

I. E. SEGAL

\title{
ERRATA, VOLUME 56
}

Electromagnetic theory. (Review by L. Brillouin.)

p. 375, line 15. For "Rutherford" read "Retherford."

Coxeter, H. S. M. The real projective plane. (Review by P. DuVal.)

p. 377, lines 2-4. The statement "but curiously it is never shown, though constantly taken for granted in the sequel, that there is always one such collineation" is in error. It has been pointed out to the author of the review that it is proved on p. 53 of the book that a collineation always exists in which one given quadrangle corresponds to another. 